\title{
Performance Analysis of Glioma Brain Tumor Segmentation using Ridgelet Transform and CANFES Methodology
}

\author{
${ }^{1}$ S.Saravanan, ${ }^{2}$ P.Thirumurugan \\ Department of Electronics and Communication Engineering, \\ PSNA College of Engineering and Technology, Tamilnadu, India. \\ Corresponding mail-saravananjask@gmail.com
}

\begin{abstract}
Objective:The Glioma brain tumor detection and segmentation methods are proposed in this paper using machine learning approaches. Methods: The boundary edge pixels are detected using Kirsch's edge detectors and then contrast adaptive histogram equalization method is applied on the edge detected pixels. Then, Ridgelet transform is applied on this enhanced brain image in order to obtain the Ridgelet multi resolution coefficients. Further, features are derived from the Ridgelet transformed coefficients and the features are optimized using Principal Component Analysis (PCA) method and these optimized features are classified into Glioma or non-Glioma brain images using Co-Active Adaptive Neuro Fuzzy Expert System (CANFES) classifier.Results: The proposed method with PCA and CANFES classification approach obtains $97.6 \%$ of se, $98.56 \%$ of sp, $98.73 \%$ of Acc, $98.85 \%$ of Pr, $98.11 \%$ of FPR and 98.185 of FNR, then the proposed Glioma brain tumor detection method using CANFES classification approach only.
\end{abstract}

Keywords: Glioma, tumors, features, Ridgelet transform, enhancement;

\section{Introductions}

The abrupt development of cells in brain and its surrounding regions forms the tumors cells. These abnormal tumor regions are classified into Glioma and Glioblastoma, which can be categorized based on the location and morphological parameters of the tumor regions in brain [710]. Glioma brain tumors are formed in the regions where brain region and spinal cord met. The cells in this junction are called as Glial cell and these cells are affected by the tumor cells. The Glial cells in this region are classified into Benign Glial cells or Malignant Glial cells, based on the damage of tissues in these regions [11]. These affected cells become tumor cells between the time periods of 8 to 12 months. The survival rate of the patient with Glioma brain tumor is about 3 years only. The Genetic disorders and tuberous sclerosis are the two main reasons for forming the Glioma brain tumors. Glioma is one type of Primary brain tumors and it leads to the sudden death in patients [12-14]. It is also coming under the category of Grade I, which develops and spreads faster. Glioblastoma is the secondary brain tumors, which may not lead to sudden death. 
This type of tumor can be called as Grade II, which develops and spreads the tumor region more slowly than Grade I. The Glioma tumors can be categorized in to the following;

- Astrocytomas;

- Oligodendrogliomas;

- Ependymomas;

Earlier stage of detection for Glioma prevents the loss of human life. Hence, there is requirement for detecting the earlier stage of the Glioma tumors. At present, craniotomy operation (opening of human head skull) is performed in the patient with Glioma tumors affected and the tumor regions are removed by surgery. The patient may extend their life period if the tumor regions are completely removed. This requires expert clinicians in order to completely detect the tumor regions. This can be only possible through proper brain image scanning techniques such as Computer Tomography (CT) or Magnetic Resonance Imaging (MRI). For the case of brain tumor detection, MRI scanning method is preferred for scanning the entire regions of the human brain. Fig.1 shows the Astrocytomas type of Glioma.

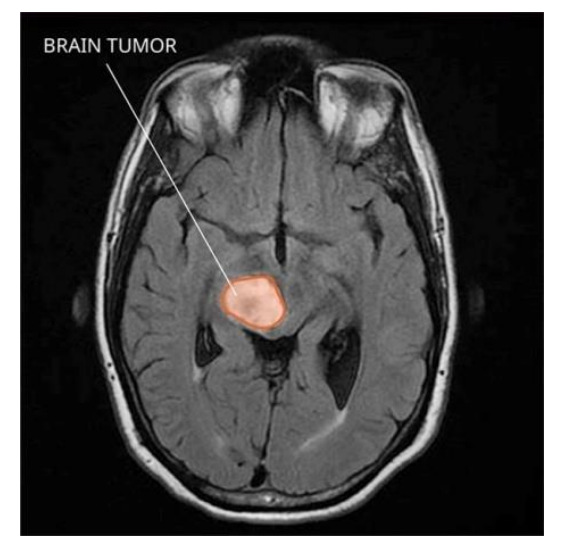

Figure 1: Glioma brain tumor brain MRI image (Astrocytomas)

\section{Literature survey}

Abdelmajid Bousselham et al. (2019) detected the tumor regions in human brain using temperature changing algorithm. The tumor contours were detected using canny edge detection method and based on these detected contour points in the source brain MRI image, thermal mapping was constructed. The gradient with large magnitude was selected from this constructed thermal map and the threshold function was applied on this mapping in order to locate the tumor regions. $80 \%$ of tumor segmentation accuracy was obtained by this method on open access brain image dataset. Rajagopal et al. (2019) detected the Glioma brain tumors using weighted random forest classification method. This method was entirely based on the parent and child node setup and split-up method. Each parent node had the center pixel in $3 * 3$ sub window mask and the intensity of the parent node pixel was split into two subsequent child nodes. These two classified child nodes were represented as Glioma case and non-Glioma case. This proposed 
method was tested by $\mathrm{k}$-fold classification testing method which yielded $0.4 \%$ of error rate when applied on the large open access dataset brain MRI images.

Farah et al. (2019) detected and removed the skull regions from the scanned brain MRI image and then elliptical area were computed from the skull removed brain MRI images. The developed brain tumor detection algorithm was applied on the large brain image dataset and the simulation results were validated by k-fold cross validation algorithm. Devkota et al. (2018) applied morphological segmentation methods on source brain MRI image in order to detect and locate the tumor pixels. The subsequent morphological operations were applied on the image and the tumor pixels were located using these mathematical operations.Kanas et al. (2015) developed intensity modeling algorithm for detecting and locating the tumor regions in brain MRI images. The authors constructed 3-dimensional random walker map from the output of the intensity modeling algorithm. Then, threshold segmentation method was applied on this constructed map. The authors obtained $94.5 \%$ of sensitivity and $95.1 \%$ of specificity on publicly available brain image dataset. Ahlgren et al. (2014) used spoiled gradient-recalled echo acquisition method to detect and segment the abnormal tumor pixels in source brain MRI images. The authors adopted fractional signal modeling to segment the abnormal tumor pixels from normal pixels. The authors obtained $92.1 \%$ of sensitivity and $93.8 \%$ of specificity on publicly available brain image dataset.

\section{Materials and Methods}

\subsection{Materials}

In this paper, the brain MRI images are obtained from 'Kaggle'- an open access dataset [15]. The brain MRI images in this dataset are having no-copyright category and many researchers are used these brain images in their brain tumor detection research. The brain images in this dataset are having $256 * 256$ pixels as its resolution by width and height. All these brain images are verified by two independent radiologists.

\subsection{Methods}

The Glioma brain tumor detection and segmentation methods are proposed in this paper using machine learning approaches. The boundary edge pixels are detected using Kirsch's edge detectors and then contrast adaptive histogram equalization method is applied on the edge detected pixels. Then, Ridgelet transform is applied on this enhanced brain image in order to obtain the Ridgelet multi resolution coefficients. Further, features are derived from the Ridgelet transformed coefficients and the features are optimized using Principal Component Analysis (PCA) method and these optimized features are classified into Glioma or non-Glioma brain images using CANFES classifier.Fig.2 shows the Glioma Brain Tumor Detection -Machine Learning Approach. 


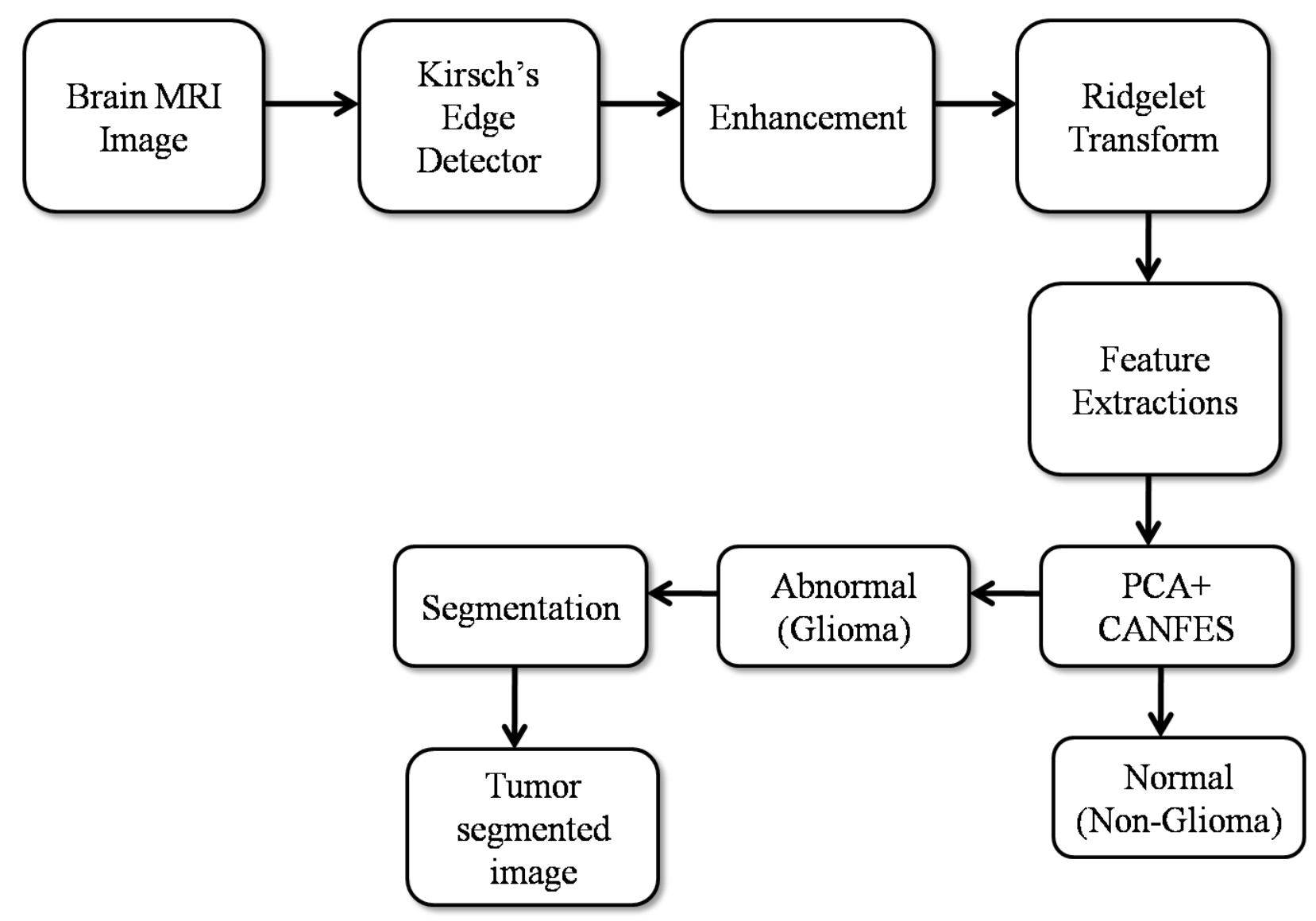

Figure 2: Glioma Brain Tumor Detection -Machine Learning Approach

\section{Enhancement}

This paper uses Kirsch'e edge detector for detecting the edge pixels in brain MRI image [16]. In conventional methods, canny and sobel edge detection methods fails to detect the edge pixels which has less pixel level intensity variations. Also, these conventional edge detectors detect the outer boundary region for every pixel in an image. In order to overcome such limitations in the conventional edge detection methods, Kirsch's edge detector is used in this paper. This Kirsch's edge detector uses eight kernels which can be operated in eight different directions along each center pixel in $3 * 3$ sub window mask. Each center pixel in $3 * 3$ sub window mask is convolved with Kirsch's edge detector kernel and the maximum pixel is detected by combining all kernel output functions together. The eight Kirsch's edge detector kernels are given in the following Table.

\section{Table 1:Kirsch's Eight Directional Templates}

\begin{tabular}{|c|c|c|c|}
\hline $\begin{array}{c}\text { Kirsch's } \\
\text { edge } \\
\text { Detector }\end{array}$ & Kernel & $\begin{array}{c}\text { Kirsch's } \\
\text { edge } \\
\text { Detector }\end{array}$ & Kernel \\
\hline
\end{tabular}




\begin{tabular}{|c|c|c|c|}
\hline $\mathrm{H} 1$ & $\begin{array}{l}{\left[\begin{array}{lll}5 & -3 & -3 \\
5 & 0 & -3 \\
5 & -3 & -3\end{array}\right] / 15}\end{array}$ & H5 & $\begin{array}{l}{\left[\begin{array}{lll}-3 & -3 & -3 \\
-3 & 0 & -3 \\
5 & 5 & 5\end{array}\right] / 15}\end{array}$ \\
\hline $\mathrm{H} 2$ & $\begin{array}{l}{\left[\begin{array}{lll}-3 & -3 & 5 \\
-3 & 0 & 5 \\
-3 & -3 & 5\end{array}\right] / 15}\end{array}$ & H6 & $\begin{array}{l}{\left[\begin{array}{lll}5 & 5 & 5 \\
-3 & 0 & -3 \\
-3 & -3 & -3\end{array}\right] / 15 ;}\end{array}$ \\
\hline H3 & $\begin{array}{l}{\left[\begin{array}{lll}-3 & -3 & -3 \\
5 & 0 & -3 \\
5 & 5 & -3\end{array}\right] / 15}\end{array}$ & $\mathrm{H} 7$ & 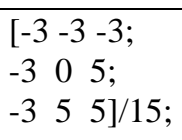 \\
\hline H4 & $\begin{array}{l}{\left[\begin{array}{lll}-3 & 5 & 5 \\
-3 & 0 & 5 \\
-3 & -3 & -3\end{array}\right] / 15}\end{array}$ & H8 & $\begin{array}{l}{\left[\begin{array}{lll}5 & 5 & -3 ; \\
5 & 0 & -3 ; \\
-3 & -3 & -3\end{array}\right] / 15 ;}\end{array}$ \\
\hline
\end{tabular}

Then, Contrast Enhanced adaptive histogram equalization method is applied now on the edge detected brain MRI image using the following equation.

$$
E(i, j)=\text { round }\left(\frac{c d f(v)-c d f \_ \text {min }}{(M * N)-c d f \_ \text {min }}\right) *(L-1)
$$

Where, $\mathrm{M}$ and $\mathrm{N}$ are the width and height of the edge detected brain MRI image and $\mathrm{L}$ is the maximum pixel intensity. Cumulative Density Function (CDF) is computed by considering the cumulative index intensity value of each pixel in $3 * 3$ sub window mask.Fig.3 (a) shows the source brain MRI image and Fig .3(b) shows itsenhanced brain MRI image.

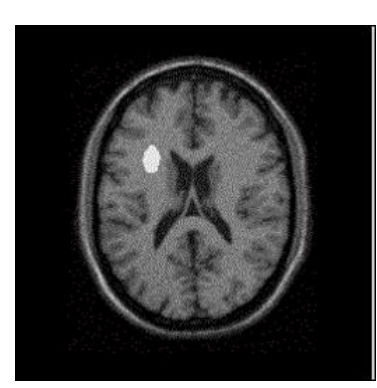

(a)

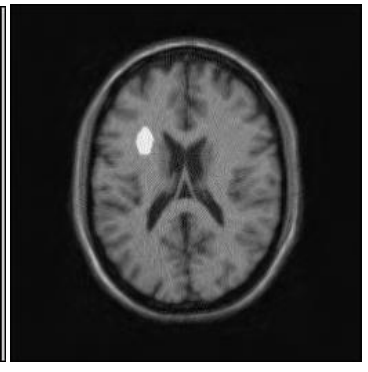

(b)

Figure 3: (a) Source brain MRI image (b) Enhanced brain MRI image

\section{Ridgelet Transform}

The pixel level transformation in an image is done through the transform which is called as Ridgelet transform [17]. There are two types of Ridgelet transforms as Discrete Ridgelet Transform (DRT) and Continuous Ridgelet Transform (CRT). The DRT performance is superior to CRT due to its non-linear effects in frequency and time domain. DRT is derived from wavelet function with respect to different lines and orientation points. It has more advantages than the wavelets. Wavelets never separate edge pixels than are belonging to smooth functions. Hence, 
wavelets cannot be used for reconstructing the sharp point edge in an image. The sparse directionality function can be achieved by Finite Ridgelet Transform (FRIT). The functional steps in this Ridgelet transform are given as,

(1). Computing the discrete radon function;

(2). Computing the wavelet transform;

The real function of this radon transform is,

$$
\begin{gathered}
R T(m, n)=\frac{1}{\sqrt{P}} \sum_{i, j \in I} I(i, j) \\
\text { Where, } I(m, n)=\left\{(i, j): j=K_{i}+l(\bmod P) ; i \in I\right\}(3)
\end{gathered}
$$

Finite Radon Transform (FRAT) is computed using FFT of the image and by through IFFT with respect to 32 different directional of pixels. Each projection in each direction delivers the transformational pixels in multi resolution format.

\section{Feature Extractions}

Features are the key parameters for differentiating the Glioma brain image from nonGlioma brain image. Features are computed from the coefficients of Ridgelet transform. In this work, the heuristic index features along with Grey Level Co-occurrence Matrix (GLCM) features are computed for identifying the tumor affected images from the normal brain MRI images.

\section{Heuristic Features}

The heuristic features relate each pixel in the source brain MRI image with its neighboring pixels in terms of its intensity distribution. The five heuristic feature sets for a each pixel $(\mathrm{x}, \mathrm{y})$ in a sub-image FI (square sized window $\mathrm{k}^{*} \mathrm{k}$ ) are given as follows,

$F_{1}(x, y)=I \quad(x, y)-\min \{F I\}$

$F_{2}(x, y)=\max \{J\}-I(x, y)$

$F_{3}(x, y)=\operatorname{abs}(I(x, y))-$ mean $\{F I\}$

$F_{4}(x, y)=\operatorname{std}\{F I\}$

$F_{5}(x, y)=I \quad(x, y)$

\section{GLCM features}

The GLCM matrix is constructed by calculating how often a pixel with grayscale intensity occurs adjacent to a pixel with the value of the source brain MRI image at different orientations as $0^{\circ}, 45^{\circ}, 90^{\circ}$ and $135^{\circ}$. In this work, GLCM matrix is constructed at $45^{\circ}$. The GLCM features are used to differentiate the malignant image from the normal brain MRI image in terms of contrast, energy, entropy and correlation, using the following equations.

$$
\begin{aligned}
& \text { Contrast }=\sum\left(|i-j|^{2} \times p(i, j)\right) \\
& \text { Energy }=\sum p(i, j)^{2}
\end{aligned}
$$


Entropy $=-\sum p(i, j)\left[\log _{2} p(i, j)\right]$

Correlation $=\sum(i-\mu i)(j-\mu j) \frac{p(i, j)}{[\sigma i . \sigma j]}$

Where, $\mathrm{p}(\mathrm{i}, \mathrm{j})$ correlates the index coordination in GLCM matrix with respect to row and column index. The mean and variance are depicted by the term $\mu$ and $\sigma$, respectively.

\section{Feature Optimization}

The extracted features are now optimized using Principal Component Analysis (PCA) optimization algorithm. In this paper, PCA algorithm is used to optimize the extracted features inorder to improve tumor segmentation accuracy. This PCA algorithm is explained in the following steps.

Step 1: Determine the population size, speed and position or coordinates of each extracted features andinitialize all these parameters for optimization.

Step 2: The population of the each extracted features can be generated by,

$$
X_{i}=\left\{x_{1}, x_{2}, x_{3} \ldots \ldots x_{N}\right\}^{T}
$$

Where as, $\mathrm{N}$ is the number of extracted features $\mathrm{s}$ in population vector or list and $\mathrm{x}$ is the particles.

Step 3: Determine the fitness value of each particles in population vector using the following equations as,

$$
f_{i}=\sum_{k=1}^{N-1}\left(x_{k}-\overline{x_{k}}\right)^{2}
$$

Where as, $\mathrm{N}$ is the number of extracted features in population vector or list and $\overline{x_{k}}$ is the mean of the population.

Step 4: Update the optimal fitness value of each individual population as Pbest.

Step 5: Update the population fitness value of each individual population as Gbest.

Step 6: The optimization metric can be estimated using the following equation as,

$$
O P T_{-} M=-\sum_{k=1}^{N}\left(\frac{f_{i}-f_{a}}{f_{i}}\right)^{2}
$$

Where as, $f_{a}$ is the average value of the fitness values.

Step 7: Update the current speed and position of each particles in population list and follow the steps from 1 to 5 . 


\section{Classifications}

The extracted features from the source test brain MRI image are classified using machine learning classification approach. Support Vector Machine (SVM) and Random Forest (RF) are known as conventional classification algorithms where the error rates of these algorithms are high. This error rate is reduced by applying Adaptive Neuro Fuzzy Inference System (ANFIS) classification algorithm which combines the benefits of both Neural Networks and Fuzzy logic. The numbers of fuzzy rules needed for each $2 * 2$ pixel combination are high and all the fuzzy rules must be executed in ANFIS classification algorithm to reach the certainty conditions. Even though the number of pixel index is null, all fuzzy rules are required for attaining the optimum classification results. These limitations are overcome by suppressing the required number of fuzzy rules for each pixel validation. Hence, Co-Active Adaptive Neuro Fuzzy Expert System (CANFES) classification algorithm is developed by integrating NN with the suppressed fuzzy rules. This also reduces the error rate during the classifications of the source test brain MRI images.

This CANFES classification architecture consists of single input layer, 3 number of hidden layers and single output layer. The input layer keeps the number of extracted features and passes this information to the next level hidden layer, which can be constructed by 15 numbers of neurons. The weight level of each neuron in each hidden layer is adaptive and its value is changed in accordance with the input extracted features. The neuron in output layer produces the output pattern by summing up all the index values which are obtained from the previous hidden layer. The output pattern from this final layer classifies the source brain MRI image into Benign (produces low index value) and Malignant (produces high index value).Fig.4 shows the CANFES architecture for Glioma brain tumor image classification.

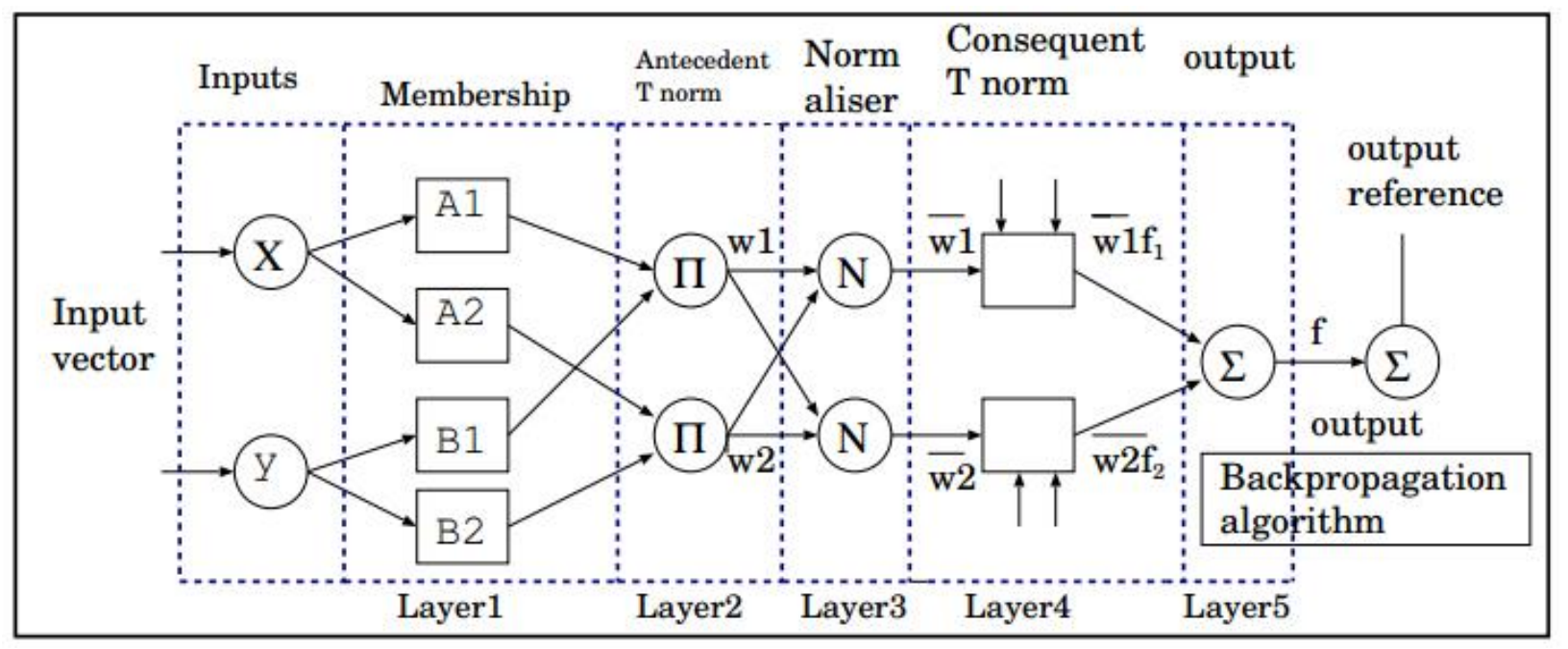

Figure 4: CANFES architecture for Glioma brain tumor image classification

\section{Segmentation}


The developed methodologies for segmentation of tumor regions in classified brain MRI image are application specific and there are no accurate common methodologies for segmenting all medical abnormal lesions in an image, with high level of segmentation accuracy. The segmentation of tumor pixels in classified brain image is complex for further diagnosis process. In order to minimize the error rate during abnormal pixel segmentation, we subject morphological opening and closing methods in this paper. The morphological opening detects and segregates the normal pixels from foreground image and places them in background. Further, morphological closing fills the removed holes in the foreground image in order to retain the image from artifacts and other noises. In this paper, disc shaped structuring element with its corresponding radius 2 is used for both opening and closing operations. The functional equations for both morphological opening and closing are explained in the following equations.

$$
\begin{aligned}
& I(\text { opened }) B=\{I 1 \in E\} ; I 1=a+b \\
& I(\text { closed }) B=\{I 2 \in E\} ; I 2+b \in I
\end{aligned}
$$

The dilation of morphological function is described as,

$$
D(I)=\sum_{k=1}^{N}\left(b_{k 1}, b_{k 2} \ldots . . b_{k n}\right) *\left(a_{k 1}, a_{k 2} \ldots \ldots a_{k n}\right)
$$

The erosion of morphological function is described as,

$$
E(I)=\prod_{k=1}^{N}\left(b_{k 1}, b_{k 2} \ldots \ldots b_{k n}\right)+\left(a_{k 1}, a_{k 2} \ldots \ldots a_{k n}\right)
$$

The dilation and erosion of the morphological functions are depicted as in the following equations.

$$
\begin{aligned}
& D_{i}(I)=\sum_{k=0}^{N-1} b_{k} * a_{k-i} \\
& E_{i}(I)=\prod_{k=0}^{N-1}\left(b_{k}+a_{k}\right)
\end{aligned}
$$

Fig.5 shows the Glioma brain tumor detected and segmented image using the proposed methodology stated in this paper.

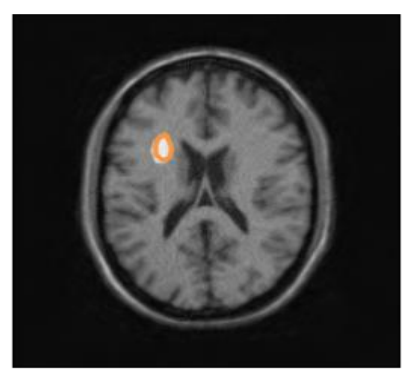

Figure 5: Tumor region segmented Glioma image 


\section{Results and Discussions}

The proposed Glioma brain tumor segmentation method is tested on the 'Kaggle'- open access dataset. The brain images in this dataset are split into sub dataset 1 and sub dataset 2 . The sub dataset1 is called as 'Learning' dataset and subdataset2 is called as 'Testing' dataset. The brain images in sub datset 1 are trained once by the methodology proposed in this paper. With the trained parameters, the images in subdataset 2 are tested using the following set of performance estimation parameters.

- Sensitivity $(\mathrm{Se}=\mathrm{TP} /(\mathrm{TP}+\mathrm{FN}))$

- Specificity $(\mathrm{Sp}=\mathrm{TN} /(\mathrm{TN}+\mathrm{FP}))$

- Accuracy $(\mathrm{Acc}=(\mathrm{TP}+\mathrm{TN}) /(\mathrm{TP}+\mathrm{FN}+\mathrm{TN}+\mathrm{FP}))$

- Precision=TP / (TP+FP)

- False positive rate $(\mathrm{FPR})=\mathrm{FP} /(\mathrm{FP}+\mathrm{TN})$

- False negative rate $(\mathrm{FNR})=\mathrm{FN} /(\mathrm{TP}+\mathrm{FN})$ approach.

Table 2shows the tumor segmentation results using proposed machine learning

Table 2: Tumor segmentation results using proposed machine learning approach

\begin{tabular}{|c|c|c|c|c|c|c|}
\hline $\begin{array}{c}\text { Brain } \\
\text { image } \\
\text { sequences }\end{array}$ & Se & Sp & Acc & Pr & FPR & FNR \\
\hline 1 & 96.5 & 98.2 & 99.5 & 98.5 & 97.1 & 97.1 \\
\hline 2 & 97.1 & 98.8 & 99.9 & 98.4 & 97.7 & 97.7 \\
\hline 3 & 98.2 & 97.1 & 98.1 & 99.7 & 98.1 & 98.8 \\
\hline 4 & 96.9 & 98.9 & 98.6 & 99.1 & 98.8 & 98.1 \\
\hline 5 & 97.7 & 99.2 & 98.4 & 99.6 & 98.6 & 98.5 \\
\hline 6 & 98.6 & 98.7 & 98.9 & 98.6 & 97.8 & 99.2 \\
\hline 7 & 97.4 & 98.6 & 98.8 & 99.1 & 98.3 & 99.1 \\
\hline 8 & 96.9 & 98.1 & 98.6 & 98.5 & 98.7 & 97.3 \\
\hline 9 & 98.6 & 99.4 & 98.4 & 98.9 & 98.5 & 97.9 \\
\hline 10 & 98.1 & 98.6 & 98.1 & 98.1 & 97.5 & 98.1 \\
\hline Average & 97.6 & 98.56 & 98.73 & 98.85 & 98.11 & 98.18 \\
\hline
\end{tabular}

Table 3 shows the analysis of proposed tumor detection system in terms of PCA and CANFES methods. The proposed method with PCA and CANFES classification approach 
obtains $97.6 \%$ of se, $98.56 \%$ of sp, $98.73 \%$ of Acc, $98.85 \%$ of Pr, $98.11 \%$ of FPR and 98.185 of FNR, then the proposed Glioma brain tumor detection method using CANFES classification approach only.

Table 3: Analysis of proposed tumor detection system in terms of PCA and CANFES methods

\begin{tabular}{|c|c|c|}
\hline \multirow{2}{*}{$\begin{array}{c}\text { Performance estimation } \\
\text { parameters } \\
(\boldsymbol{\%})\end{array}$} & CANFES only & Pethodology \\
\cline { 2 - 3 } & & 94.1 \\
\hline Se & 95.67 & 97.6 \\
\hline Acc & 96.28 & 98.56 \\
\hline Pr & 96.85 & 98.73 \\
\hline FPR & 95.19 & 98.85 \\
\hline FNR & 95.29 & 98.11 \\
\hline
\end{tabular}

Table 4 shows the comparisons of proposed Glioma tumor detection with conventional methods as Abdelmajid Bousselham et al. (2019), Rajagopal et al. (2019) and Devkota et al. (2018). From Table 3, it is very clear that the proposed Glioma brain tumor detection and segmentation method using machine learning approach provides superior results while compared with other conventional Glioma brain tumor detection methods.

Table 4: Comparisons of proposed Glioma tumor detection with conventional methods

\begin{tabular}{|c|c|c|c|c|}
\hline Methodologies & $\begin{array}{c}\text { Se } \\
(\%)\end{array}$ & $\begin{array}{c}\text { Sp } \\
(\%)\end{array}$ & $\begin{array}{c}\text { Acc } \\
(\%)\end{array}$ & $\begin{array}{c}\text { Pr } \\
(\%)\end{array}$ \\
\hline Proposed work & $\mathbf{9 7 . 6}$ & $\mathbf{9 8 . 5 6}$ & $\mathbf{9 8 . 7 3}$ & $\mathbf{9 8 . 8 5}$ \\
\hline $\begin{array}{c}\text { Abdelmajid } \\
\text { Bousselham et al. } \\
\text { (2019) }\end{array}$ & 95.1 & 96.97 & 96.95 & 97.57 \\
\hline Rajagopalet al. (2019) & 94.9 & 95.20 & 96.27 & 97.18 \\
\hline Devkota et al. (2018) & 94.8 & 95.19 & 95.96 & 96.75 \\
\hline
\end{tabular}

\section{Conclusions}

The machine learning classification approach based brain tumor detection and segmentation method is proposed in this paper. The proposed methods have Enhancement using Kirsch's edge detectors, Ridgelet transform, features extraction, CANFES classification and morphological segmentation modules. The functional operations of each proposed methods are explained in this paper. The proposed method with PCA and CANFES classification approach obtains $97.6 \%$ of se, $98.56 \%$ of sp, $98.73 \%$ of Acc, $98.85 \%$ of Pr, $98.11 \%$ of FPR and 98.185 of 
FNR, then the proposed Glioma brain tumor detection method using CANFES classification approach only. It is very clear that the proposed Glioma brain tumor detection and segmentation method using machine learning approach provides superior results while compared with other conventional Glioma brain tumor detection methods.

\section{References}

[1] Abdelmajid Bousselham , Omar Bouattane, Mohamed Youssfi, and Abdelhadi Raihani, "Towards Reinforced Brain Tumor Segmentation on MRI Images Based on Temperature Changes on Pathologic Area", Hindawi International Journal of Biomedical Imaging, Volume 2019, Article ID 1758948.

[2] V. G. Kanas, E. I. Zacharaki, C. Davatzikos, K. N. Sgarbas, and V. Megalooikonomou, "A low cost approach for brain tumor segmentation based on intensity modeling and 3D Random Walker,” Biomedical Signal Processing and Control, vol. 22, pp. 19-30, 2015.

[3] Ahlgren, R. Wirestam, F. Ståhlberg, and L. Knutsson, "Automatic brain segmentation using fractional signal modeling of a multiple flip angle, spoiled gradient-recalled echo acquisition," Magnetic Resonance Materials in Physics, Biology and Medicine, vol. 27, no. 6, pp. 551-565, 2014.

[4] R. Rajagopal, "Glioma brain tumor detection and segmentation using weighting random forest classifier with optimized ant colony features", International Journal of Imaging systems and Technology, volume29, Issue3, 2019, Pages 353-359.

[5] Farah Q. Al-khalidi, Maha A. Bayati and Shaimaa H. Alkinany, 2019. Tumor Detection and Extraction in the Human Brain. Journal of Engineering and Applied Sciences, 14: 2333-2339.

[6] Devkota, B., Alsadoon, A., Prasad, P. W. C., Singh, A. K., \& Elchouemi, A. (2018). Image Segmentation for Early Stage Brain Tumor Detection using Mathematical Morphological Reconstruction. Procedia Computer Science, 125, 115-123.

[7] Hemanth, D. J., \& Anitha, J. (2012) "Image Pre-processing and Feature Extraction Techniques for Magnetic Resonance Brain Image Analysis," Computer Applications for Communication, Networking, and Digital Contents 349-356.

[8] Nabizadeh,N., \& Kubat,M. (2015) "Brain tumors detection and segmentation in MR images: Gabor wavelet vs. statistical features," Computers \& Electrical Engineering, 45:286-301.

[9] L. Guo, L. Zhao, Y. Wu, Y. Li, G. Xu, and Q. Yan, "Tumor detection in MR images using one-class immune feature weighted SVMs," IEEE Transactions on Magnetics, vol. 47, no. 10, pp. 3849-3852, 2011.

[10] Y. Kong, Y. Deng, and Q. Dai, "Discriminative clustering and feature selection for brain MRI segmentation," IEEE Signal Processing Letters, vol. 22, no. 5, pp. 573$577,2015$. 
[11] Q. Ain, M. A. Jaffar, and T.-S. Choi, "Fuzzy anisotropic diffusion based segmentation and texture based ensemble classification of brain tumor," Applied Soft Computing Journal, vol. 21, pp. 330-340, 2014.

[12] W. Cui, Y. Wang, Y. Fan, Y. Feng, and T. Lei, "Localized FCM clustering with spatial information for medical image segmentation and bias field estimation," International Journal of Biomedical Imaging, vol. 2013, Article ID 930301, 8 pages, 2013.

[13] S. Lal and M. Chandra, "Efficient algorithm for contrast enhancement of natural images," International Arab Journal of Information Technology, vol. 11, no. 1, pp. 95102, 2014.

[14] Parveen and A. Singh, "Detection of brain tumor in MRI images, using combination of fuzzy c-means and SVM," in Proceedings of the 2nd International Conference on Signal Processing and Integrated Networks (SPIN '15), pp. 98-102, February 2015.

[15] https://www.kaggle.com/navoneel/brain-mri-images-for-brain-tumor-detection.

[16] A.R. Venmathi, E.N. Ganesh and N. Kumaratharan,'Kirsch Compass Kernel Edge Detection Algorithm for Micro Calcification Clusters in Mammograms",MiddleEast J. Sci. Res., 24 (4): 1530-1535, 2016.

[17] Lakshmanan Akila and Rajakumar Roopkumar, "Ridgelet transform for quarternion-valued functions",International Journal of Wavelets, Multiresolution and Information ProcessingVol. 14, No. 01, 1650006 (2016). 